\title{
On Generalized Tetranacci Numbers: Closed Form Formulas of the Sum $\sum_{k=0}^{n} W_{k}^{2}$ of the Squares of Terms
}

\author{
Yüksel Soykan \\ Department of Mathematics, \\ Art and Science Faculty, \\ Zonguldak Bülent Ecevit University, \\ 67100, Zonguldak, Turkey \\ e-mail: yuksel_soykan@hotmail.com
}

Abstract. In this paper, closed forms of the sum formulas $\sum_{k=0}^{n} W_{k}^{2}$ for the squares of generalized Tetranacci numbers are presented. We also present the sum formulas $\sum_{k=0}^{n} W_{k+1} W_{k}, \sum_{k=0}^{n} W_{k+2} W_{k}$, and $\sum_{k=0}^{n} W_{k+3} W_{k}$. As special cases, we give summation formulas of the of Tetranacci, Tetranacci-Lucas and some other fourth order linear recurrance sequences.

2010 Mathematics Subject Classification. 11B39, 11B83.

Keywords. Sum of squares, fourth order recurrence, Tetranacci numbers, Tetranacci-Lucas numbers.

\section{Introduction}

There have been so many studies of the sequences of numbers in the literature which are defined recursively. Two of these type of sequences are the sequences of Tetranacci and Tetranacci-Lucas which are special case of generalized Tetranacci numbers. A generalized Tetranacci sequence $\left\{W_{n}\right\}_{n \geq 0}=\left\{W_{n}\left(W_{0}, W_{1}, W_{2}, W_{3}\right)\right\}_{n \geq 0}$ is defined by the fourth-order recurrence relations

$$
W_{n}=r W_{n-1}+s W_{n-2}+t W_{n-3}+u W_{n-4}, \quad W_{0}=a, W_{1}=b, W_{2}=c, W_{3}=d, \quad n \geq 4
$$

with the initial values $W_{0}=c_{0}, W_{1}=c_{1}, W_{2}=c_{2}, W_{3}=c_{3}$ not all being zero.

This sequence has been studied by many authors and more detail can be found in the extensive literature dedicated to these sequences, see for example $[7,10,11,16,25,26]$.

The sequence $\left\{W_{n}\right\}_{n \geq 0}$ can be extended to negative subscripts by defining

$$
W_{-n}=-\frac{t}{u} W_{-(n-1)}-\frac{s}{u} W_{-(n-2)}-\frac{r}{u} W_{-(n-3)}+\frac{1}{u} W_{-(n-4)}
$$

for $n=1,2,3, \ldots$. Therefore, recurrence (1.1) holds for all integer $n$. 
In literature, for example, the following names and notations (see Table 1) are used for the special case of $r, s, t, u$ and initial values.

Table 1 A few special case of generalized Tetranacci sequences.

\begin{tabular}{ccc}
\hline Sequences (Numbers) & Notation & OEIS [24] \\
\hline Tetranacci & $\left\{M_{n}\right\}=\left\{W_{n}(0,1,1,2 ; 1,1,1,1)\right\}$ & A000078 \\
Tetranacci-Lucas & $\left\{R_{n}\right\}=\left\{W_{n}(4,1,3,7 ; 1,1,1,1)\right\}$ & A073817 \\
fourth order Pell & $\left\{P_{n}^{(4)}\right\}=\left\{W_{n}(0,1,2,5 ; 2,1,1,1)\right\}$ & A103142 \\
fourth order Pell-Lucas & $\left\{Q_{n}^{(4)}\right\}=\left\{W_{n}(4,2,6,17 ; 2,1,1,1)\right\}$ & A331413 \\
modified fourth order Pell & $\left\{E_{n}^{(4)}\right\}=\left\{W_{n}(0,1,1,3 ; 2,1,1,1)\right\}$ & A190139 \\
4-primes & $\left\{G_{n}\right\}=\left\{W_{n}(0,0,1,2 ; 2,3,5,7)\right\}$ & \\
Lucas 4-primes & $\left\{H_{n}\right\}=\left\{W_{n}(4,2,10,41 ; 2,3,5,7)\right\}$ & \\
modified 4-primes & $\left\{E_{n}\right\}=\left\{W_{n}(0,0,1,1 ; 2,3,5,7)\right\}$ & \\
\hline
\end{tabular}

Here OEIS stands for On-line Encyclopedia of Integer Sequences. In the rest of the paper, for easy writing, we drop the superscripts and write $P_{n}, Q_{n}$ and $E_{n}$ for $P_{n}^{(4)}, Q_{n}^{(4)}$ and $E_{n}^{(4)}$, respectively. For generalized fourth order Pell numbers and generalized 4-primes numbers see [17] and [23], respectively.

The evaluation of sums of powers of these sequences is a challenging issue. Two pretty examples are

$\sum_{k=0}^{n} R_{k}^{2}=\frac{1}{3}\left(-R_{n+4}^{2}-3 R_{n+3}^{2}-4 R_{n+2}^{2}-4 R_{n+1}^{2}+3 R_{n+4} R_{n+3}+2 R_{n+4} R_{n+2}+R_{n+4} R_{n+1}-R_{n+2} R_{n+1}+43\right)$

and

$\sum_{k=0}^{n} P_{k}^{2}=\frac{1}{56}\left(-9 P_{n+4}^{2}-57 P_{n+3}^{2}-68 P_{n+2}^{2}-65 P_{n+1}^{2}+42 P_{n+4} P_{n+3}+20 P_{n+4} P_{n+2}+6 P_{n+4} P_{n+1}-\right.$ $\left.4 P_{n+3} P_{n+2}+2 P_{n+3} P_{n+1}-12 P_{n+2} P_{n+1}+9\right)$.

In [15], the author showed that

$$
\sum_{k=0}^{n} M_{k}^{2}=\frac{1}{3}\left(1+3 M_{n} M_{n+1}-\left(M_{n+1}-M_{n-1}\right)^{2}+M_{n} M_{n-2}+M_{n-2} M_{n-3}\right)
$$

by using induction with a (long) proof. In this paper, as an easy corollary to our main result, we find that $(1.3)$

$\sum_{k=0}^{n} M_{k}^{2}=\frac{1}{3}\left(-M_{n+4}^{2}-3 M_{n+3}^{2}-4 M_{n+2}^{2}-4 M_{n+1}^{2}+3 M_{n+4} M_{n+3}+2 M_{n+4} M_{n+2}+M_{n+4} M_{n+1}-M_{n+2} M_{n+1}+1\right)$.

By using Binet formula of Tetranacci numbers (or the fourth-order recurrence relations (1.1)) it can be seen that the rights sides of the formulas (1.2) and (1.3) are equal.

In this work, we derive expressions for sums of second powers of generalized Tetranacci numbers. We present some works on sum formulas of powers of the numbers in the following Table 2.

Table 2. A few special study on sum formulas of second, third and arbitrary powers.

\begin{tabular}{cccc}
\hline Name of sequence & sums of second powers & sums of third powers & sums of powers \\
\hline Generalized Fibonacci & {$[1,2,6,8,9,18]$} & {$[5,19,21,22,27]$} & {$[3,4,12]$} \\
Generalized Tribonacci & {$[14,20]$} & & \\
Generalized Tetranacci & {$[13,15]$} & & \\
\hline
\end{tabular}




\section{Main Result}

Let

$$
\Delta=(r-s+t-u+1)\left(s+u+r^{2} u+s u^{2}+r t+2 s u-t^{2}-u^{2}-u^{3}-r t u+1\right)(r+s+t+u-1) .
$$

Theorem 2.1. If $\Delta \neq 0$ then

(a):

(b):

$$
\sum_{k=0}^{n} W_{k}^{2}=\frac{\Delta_{1}}{\Delta}
$$

(c):

(d):

$$
\sum_{k=0}^{n} W_{k+1} W_{k}=\frac{\Delta_{2}}{\Delta}
$$

$$
\sum_{k=0}^{n} W_{k+2} W_{k}=\frac{\Delta_{3}}{\Delta}
$$

(d):

$$
\sum_{k=0}^{n} W_{k+3} W_{k}=\frac{\Delta_{4}}{\Delta}
$$

where

$$
\Delta_{1}=\sum_{k=1}^{20} \Gamma_{k}, \Delta_{2}=\sum_{k=1}^{20} \Theta_{k}, \Delta_{3}=\sum_{k=1}^{20} \Phi_{k}, \Delta_{4}=\sum_{k=1}^{20} \Psi_{k}
$$

with

$$
\begin{aligned}
& \Gamma_{1}=-\left(s+u+r^{2} u-s u^{2}+r t+t^{2}+u^{2}-u^{3}+r t u-1\right) W_{n+4}^{2}, \\
& \Gamma_{2}=-\left(s+u+r^{2} s+r^{3} t-s u^{2}+r^{4} u+r^{2} t^{2}+r^{2} u^{2}-r^{2} u^{3}+r t+r^{2}+t^{2}+u^{2}-u^{3}+2 r^{2} s u-2 r t u^{2}+\right. \\
& \left.r^{3} t u-r^{2} s u^{2}+2 r s t+3 r t u-1\right) W_{n+3}^{2}, \\
& \Gamma_{3}=-\left(r^{4} u+r^{3} t u+r^{3} t-r^{2} s^{2} u-r^{2} s u^{2}+4 r^{2} s u+r^{2} s+r^{2} t^{2}-r^{2} u^{3}+r^{2} u^{2}+r^{2}+r s^{2} t u-r s^{2}\right. \\
& t+2 r s t u^{2}+4 r s t-2 r t u^{2}+3 r t u+r t-s^{3} u^{2}-2 s^{3} u-s^{3}+s^{2} t^{2}-s^{2} u^{3}-s^{2} u^{2}+s^{2} u+s^{2}+2 \\
& \left.s t^{2} u-s u^{2}+s+t^{2}-u^{3}+u^{2}+u-1\right) W_{n+2}^{2}, \\
& \Gamma_{4}=-\left(r^{4} u+r^{3} t u+r^{3} t-r^{2} s^{2} u-r^{2} s u^{2}+4 r^{2} s u+r^{2} s-r^{2} t^{2} u+r^{2} t^{2}-r^{2} u^{3}+r^{2} u^{2}+r^{2}+r s^{2}\right. \\
& t u-r s^{2} t+4 r s t u^{2}+4 r s t-r t^{3} u-r t^{3}-4 r t u^{2}+5 r t u+r t-s^{3} u^{2}-2 s^{3} u-s^{3}+s^{2} t^{2}-s^{2} u^{3}-s^{2} u^{2}+ \\
& \left.s^{2} u+s^{2}+s t^{2} u^{2}+4 s t^{2} u-s t^{2}-s u^{2}+s-t^{4}-t^{2} u^{3}+t^{2} u^{2}-t^{2} u+2 t^{2}-u^{3}+u^{2}+u-1\right) W_{n+1}^{2}, \\
& \Gamma_{5}=2\left(r t^{2}+r^{2} t+r u^{2}-r u^{3}+r^{3} u-t u^{2}+r s+s t+t u-r s u^{2}+r^{2} t u+r s u\right) W_{n+4} W_{n+3}, \\
& \Gamma_{6}=2\left(r^{2} u+r s t u+r t u^{2}+r t-s^{2} u^{2}-s^{2} u+s t^{2}-s u^{3}+s u+t^{2} u\right) W_{n+4} W_{n+2}, \\
& \Gamma_{7}=2 u\left(r-t u^{2}-r u+s t+t u+r s u\right) W_{n+4} W_{n+1}, \\
& \Gamma_{8}=-2\left(r^{2} s t u+r^{2} t u^{2}-r^{2} t u-r s^{2} u^{2}+r s t^{2}-r s u^{3}+r s u^{2}-r s u+r t^{2} u-r t^{2}+r u^{3}-r u^{2}+s^{2}\right. \\
& \left.t-s t u^{2}+s t u-s t+t u^{2}-t u\right) W_{n+3} W_{n+2}, \\
& \Gamma_{9}=-2 u\left(r^{2} s u-r^{2} u+r s t-r t u^{2}-r t+s^{2} u+s^{2}+s u^{2}-s-t^{2} u\right) W_{n+3} W_{n+1}, \\
& \Gamma_{10}=-2 u\left(r t^{2}-t+r u^{2}-r u+s t+t u+t^{3}-r s u^{2}+r t^{2} u+r^{2} t u-s t u^{2}-s t u\right) W_{n+2} W_{n+1}, \\
& \Gamma_{11}=\left(s+u+r^{2} u-s u^{2}+r t+t^{2}+u^{2}-u^{3}+r t u-1\right) W_{3}^{2},
\end{aligned}
$$


$\Gamma_{12}=\left(s+u+r^{2} s+r^{3} t-s u^{2}+r^{4} u+r^{2} t^{2}+r^{2} u^{2}-r^{2} u^{3}+r t+r^{2}+t^{2}+u^{2}-u^{3}+2 r^{2} s u-2 r t u^{2}+\right.$ $\left.r^{3} t u-r^{2} s u^{2}+2 r s t+3 r t u-1\right) W_{2}^{2}$,

$\Gamma_{13}=\left(r^{4} u+r^{3} t u+r^{3} t-r^{2} s^{2} u-r^{2} s u^{2}+4 r^{2} s u+r^{2} s+r^{2} t^{2}-r^{2} u^{3}+r^{2} u^{2}+r^{2}+r s^{2} t u-r s^{2}\right.$ $t+2 r s t u^{2}+4 r s t-2 r t u^{2}+3 r t u+r t-s^{3} u^{2}-2 s^{3} u-s^{3}+s^{2} t^{2}-s^{2} u^{3}-s^{2} u^{2}+s^{2} u+s^{2}+2$ $\left.s t^{2} u-s u^{2}+s+t^{2}-u^{3}+u^{2}+u-1\right) W_{1}^{2}$,

$\Gamma_{14}=\left(r^{4} u+r^{3} t u+r^{3} t-r^{2} s^{2} u-r^{2} s u^{2}+4 r^{2} s u+r^{2} s-r^{2} t^{2} u+r^{2} t^{2}-r^{2} u^{3}+r^{2} u^{2}+r^{2}+r s^{2}\right.$ $t u-r s^{2} t+4 r s t u^{2}+4 r s t-r t^{3} u-r t^{3}-4 r t u^{2}+5 r t u+r t-s^{3} u^{2}-2 s^{3} u-s^{3}+s^{2} t^{2}-s^{2} u^{3}-s^{2} u^{2}+$ $\left.s^{2} u+s^{2}+s t^{2} u^{2}+4 s t^{2} u-s t^{2}-s u^{2}+s-t^{4}-t^{2} u^{3}+t^{2} u^{2}-t^{2} u+2 t^{2}-u^{3}+u^{2}+u-1\right) W_{0}^{2}$,

$\Gamma_{15}=-2\left(r t^{2}+r^{2} t+r u^{2}-r u^{3}+r^{3} u-t u^{2}+r s+s t+t u-r s u^{2}+r^{2} t u+r s u\right) W_{3} W_{2}$,

$\Gamma_{16}=-2\left(r^{2} u+r s t u+r t u^{2}+r t-s^{2} u^{2}-s^{2} u+s t^{2}-s u^{3}+s u+t^{2} u\right) W_{3} W_{1}$,

$\Gamma_{17}=2\left(r^{2} s t u+r^{2} t u^{2}-r^{2} t u-r s^{2} u^{2}+r s t^{2}-r s u^{3}+r s u^{2}-r s u+r t^{2} u-r t^{2}+r u^{3}-r u^{2}+s^{2}\right.$ $\left.t-s t u^{2}+s t u-s t+t u^{2}-t u\right) W_{2} W_{1}$,

$\Gamma_{18}=-2 u\left(r-t u^{2}-r u+s t+t u+r s u\right) W_{3} W_{0}$,

$\Gamma_{19}=2 u\left(r^{2} s u-r^{2} u+r s t-r t u^{2}-r t+s^{2} u+s^{2}+s u^{2}-s-t^{2} u\right) W_{2} W_{0}$,

$\Gamma_{20}=2 u\left(r t^{2}-t+r u^{2}-r u+s t+t u+t^{3}-r s u^{2}+r t^{2} u+r^{2} t u-s t u^{2}-s t u\right) W_{1} W_{0}$,

and

$\Theta_{1}=\left(r-t u^{2}-r u+s t+t u+r s u\right) W_{n+4}^{2}$,

$\Theta_{2}=\left(r^{3} s u+r^{2} s t-r^{2} t u^{2}+r^{2} t u+r s^{2} u+r s^{2}+r s u-r t^{2} u+r t^{2}-r u^{3}+r u^{2}+s t-t u^{2}+t u\right)$ $W_{n+3}^{2}$,

$\Theta_{3}=\left(-r^{2} t u^{2}+r^{2} t u+r s t^{2} u+r s u^{3}-r t^{2} u+r t^{2}-r u^{3}+r u^{2}-s^{2} t u^{2}-s^{2} t u+s t^{3}+s t u^{2}-t u^{2}+\right.$ tu) $W_{n+2}^{2}$,

$\Theta_{4}=u^{2}\left(r-t u^{2}-r u+s t+t u+r s u\right) W_{n+1}^{2}$,

$\Theta_{5}=-\left(2 r^{2} s u-r^{2} u+r^{2}+2 r s t-2 r t u^{2}+2 r t u+s^{2} u+s^{2}-t^{2} u+t^{2}-u^{3}+u^{2}+u-1\right) W_{n+4} W_{n+3}$,

$\Theta_{6}=\left(r^{3} u+r^{2} t-r s^{2} u+2 r s u-r t^{2} u-r u^{3}+r u-s^{2} t+2 s t u^{2}-t^{3}-t u^{2}+t\right) W_{n+4} W_{n+2}$,

$\Theta_{7}=u\left(-r^{2} u+r^{2}-s^{2} u-s^{2}+t^{2} u-t^{2}+u^{3}-u^{2}-u+1\right) W_{n+4} W_{n+1}$,

$\Theta_{8}=-\left(r^{4} u+r^{3} t-r^{2} s^{2} u+3 r^{2} s u+r^{2} s-r^{2} t^{2} u-r^{2} u^{3}+r^{2}-r s^{2} t+2 r s t u^{2}+2 r s t-r t^{3}-3 r\right.$ $t u^{2}+2 r t u+r t-s^{3} u-s^{3}+s^{2} u+s^{2}+s t^{2} u-s t^{2}+s u^{3}-s u^{2}-s u+s-t^{2} u+t^{2}-u^{3}+u^{2}+u-$ 1) $W_{n+3} W_{n+2}$,

$\Theta_{9}=u\left(r^{3} u+r^{2} t+r s^{2} u+2 r s-r t^{2} u-r u^{3}+r u+s^{2} t+2 s t u-t^{3}-t u^{2}+t\right) W_{n+3} W_{n+1}$,

$\Theta_{10}=-\left(r^{4} u+r^{3} t u+r^{3} t-r^{2} s^{2} u-r^{2} s u^{2}+4 r^{2} s u+r^{2} s-r^{2} t^{2} u+r^{2} t^{2}-r^{2} u^{3}+r^{2}+r s^{2} t u-r s^{2}\right.$ $t+2 r s t u^{2}+4 r s t-r t^{3} u-r t^{3}-r t u^{3}-3 r t u^{2}+3 r t u+r t-s^{3} u^{2}-2 s^{3} u-s^{3}+s^{2} t^{2}+s^{2} u+s^{2}+$ $\left.s t^{2} u^{2}+2 s t^{2} u-s t^{2}+s u^{4}-2 s u^{2}+s-t^{4}-t^{2} u^{2}-t^{2} u+2 t^{2}-u^{3}+u^{2}+u-1\right) W_{n+2} W_{n+1}$,

$\Theta_{11}=-\left(r-t u^{2}-r u+s t+t u+r s u\right) W_{3}^{2}$,

$\Theta_{12}=-\left(r^{3} s u+r^{2} s t-r^{2} t u^{2}+r^{2} t u+r s^{2} u+r s^{2}+r s u-r t^{2} u+r t^{2}-r u^{3}+r u^{2}+s t-t u^{2}+t u\right)$ $W_{2}^{2}$, 
$\Theta_{13}=\left(r^{2} t u^{2}-r^{2} t u-r s t^{2} u-r s u^{3}+r t^{2} u-r t^{2}+r u^{3}-r u^{2}+s^{2} t u^{2}+s^{2} t u-s t^{3}-s t u^{2}+t u^{2}-\right.$ tu) $W_{1}^{2}$,

$\Theta_{14}=-u^{2}\left(r-t u^{2}-r u+s t+t u+r s u\right) W_{0}^{2}$,

$\Theta_{15}=\left(2 r^{2} s u-r^{2} u+r^{2}+2 r s t-2 r t u^{2}+2 r t u+s^{2} u+s^{2}-t^{2} u+t^{2}-u^{3}+u^{2}+u-1\right) W_{3} W_{2}$,

$\Theta_{16}=-\left(r^{3} u+r^{2} t-r s^{2} u+2 r s u-r t^{2} u-r u^{3}+r u-s^{2} t+2 s t u^{2}-t^{3}-t u^{2}+t\right) W_{3} W_{1}$,

$\Theta_{17}=\left(r^{4} u+r^{3} t-r^{2} s^{2} u+3 r^{2} s u+r^{2} s-r^{2} t^{2} u-r^{2} u^{3}+r^{2}-r s^{2} t+2 r s t u^{2}+2 r s t-r t^{3}-3 r\right.$ $\left.t u^{2}+2 r t u+r t-s^{3} u-s^{3}+s^{2} u+s^{2}+s t^{2} u-s t^{2}+s u^{3}-s u^{2}-s u+s-t^{2} u+t^{2}-u^{3}+u^{2}+u-1\right)$ $W_{2} W_{1}$,

$\Theta_{18}=u\left(r^{2} u-r^{2}+s^{2} u+s^{2}-t^{2} u+t^{2}-u^{3}+u^{2}+u-1\right) W_{3} W_{0}$,

$\Theta_{19}=-u\left(r^{3} u+r^{2} t+r s^{2} u+2 r s-r t^{2} u-r u^{3}+r u+s^{2} t+2 s t u-t^{3}-t u^{2}+t\right) W_{2} W_{0}$,

$\Theta_{20}=\left(r^{4} u+r^{3} t u+r^{3} t-r^{2} s^{2} u-r^{2} s u^{2}+4 r^{2} s u+r^{2} s-r^{2} t^{2} u+r^{2} t^{2}-r^{2} u^{3}+r^{2}+r s^{2} t u-r s^{2}\right.$ $t+2 r s t u^{2}+4 r s t-r t^{3} u-r t^{3}-r t u^{3}-3 r t u^{2}+3 r t u+r t-s^{3} u^{2}-2 s^{3} u-s^{3}+s^{2} t^{2}+s^{2} u+s^{2}+$ $\left.s t^{2} u^{2}+2 s t^{2} u-s t^{2}+s u^{4}-2 s u^{2}+s-t^{4}-t^{2} u^{2}-t^{2} u+2 t^{2}-u^{3}+u^{2}+u-1\right) W_{1} W_{0}$,

and

$\Phi_{1}=\left(r^{2}+r t u+r t-s^{2} u-s^{2}-s u^{2}+s+t^{2} u\right) W_{n+4}^{2}$,

$\Phi_{2}=\left(r^{3} t u-r^{2} s^{2} u-r^{2} s u^{2}-r^{2} s+r^{2} t^{2} u+r^{2} t^{2}+r^{2} u^{2}-r s^{2} t-2 r s t u+r t^{3}+r t u^{2}+r t u-s^{2}\right.$ $\left.u-s^{2}-s u^{2}+s+t^{2} u\right) W_{n+3}^{2}$,

$\Phi_{3}=\left(r^{3} t u+r^{2} t^{2} u+r^{2} t^{2}+r^{2} u^{2}-r s^{2} t u+2 r s t u+r t^{3}+r t u^{2}+r t u-s^{2} t^{2}-s^{2} u^{3}-s^{2} u^{2}+\right.$ $\left.s t^{2} u^{2}+s t^{2}-s u^{4}+s u^{2}+t^{2} u\right) W_{n+2}^{2}$,

$\Phi_{4}=u^{2}\left(r^{2}+r t u+r t-s^{2} u-s^{2}-s u^{2}+s+t^{2} u\right) W_{n+1}^{2}$,

$\Phi_{5}=\left(-r^{3}-2 r^{2} t u-r^{2} t+2 r s^{2} u+r s^{2}+2 r s u^{2}-2 r t^{2} u-r t^{2}-r u^{2}+r+s^{2} t+2 s t u-t^{3}-t u^{2}+\right.$ t) $W_{n+4} W_{n+3}$,

$\Phi_{6}=-\left(r^{2} s u+r^{2} s+r^{2} u^{2}+r^{2}+2 r s t+4 r t u-s^{3} u-s^{3}-s^{2} u^{2}+s^{2}+s t^{2} u-s t^{2}-s u^{3}-s u^{2}+s u+\right.$ $\left.s+t^{2} u^{2}+t^{2}-u^{4}+2 u^{2}-1\right) W_{n+4} W_{n+2}$,

$\Phi_{7}=u\left(r^{3}+r^{2} t-r s^{2}+2 r s-r t^{2}-r u^{2}+r+s^{2} t+2 s t u-t^{3}-t u^{2}+t\right) W_{n+4} W_{n+1}$,

$\Phi_{8}=\left(r^{3} s u+r^{3} u^{2}+r^{2} s t+2 r^{2} t u-r^{2} t-r s^{3} u-r s^{2} u^{2}+2 r s^{2} u+r s t^{2} u-r s u^{3}+2 r s u^{2}+r s\right.$ $\left.u+r t^{2} u^{2}-2 r t^{2} u-r u^{4}+r u^{2}-s^{3} t-2 s^{2} t u+s^{2} t+s t^{3}+s t u^{2}+2 s t u-s t-t^{3}-t u^{2}+t\right) W_{n+3} W_{n+2}$,

$\Phi_{9}=-\left(r^{4} u+r^{3} t u+r^{3} t-r^{2} s^{2} u+3 r^{2} s u+r^{2} s-r^{2} t^{2} u+r^{2} t^{2}-r^{2} u^{3}+r^{2} u^{2}+r^{2} u+r^{2}+r s^{2} t u-\right.$ $r s^{2} t+2 r s t u^{2}+4 r s t-r t^{3} u-r t^{3}-r t u^{3}-r t u^{2}+5 r t u+r t-s^{3} u-s^{3}+s^{2} t^{2}-s^{2} u^{2}+s^{2}+$ $\left.3 s t^{2} u-s t^{2}-s u^{3}-s u^{2}+s u+s-t^{4}+2 t^{2}-u^{4}+2 u^{2}-1\right) W_{n+3} W_{n+1}$,

$\Phi_{10}=u\left(r^{3} u+r^{2} t u+2 r^{2} t-r s^{2} u+2 r s u+r t^{2} u+2 r t^{2}-r u^{3}+r u-s^{2} t u-2 s^{2} t+2 s t+t^{3} u-t u^{3}+\right.$ tu) $W_{n+2} W_{n+1}$,

$\Phi_{11}=-\left(r^{2}+r t u+r t-s^{2} u-s^{2}-s u^{2}+s+t^{2} u\right) W_{3}^{2}$,

$\Phi_{12}=-\left(r^{3} t u-r^{2} s^{2} u-r^{2} s u^{2}-r^{2} s+r^{2} t^{2} u+r^{2} t^{2}+r^{2} u^{2}-r s^{2} t-2 r s t u+r t^{3}+r t u^{2}+r t u-s^{2}\right.$ $\left.u-s^{2}-s u^{2}+s+t^{2} u\right) W_{2}^{2}$, 
$\Phi_{13}=-\left(r^{3} t u+r^{2} t^{2} u+r^{2} t^{2}+r^{2} u^{2}-r s^{2} t u+2 r s t u+r t^{3}+r t u^{2}+r t u-s^{2} t^{2}-s^{2} u^{3}-s^{2} u^{2}+\right.$ $\left.s t^{2} u^{2}+s t^{2}-s u^{4}+s u^{2}+t^{2} u\right) W_{1}^{2}$,

$\Phi_{14}=-u^{2}\left(r^{2}+r t u+r t-s^{2} u-s^{2}-s u^{2}+s+t^{2} u\right) W_{0}^{2}$,

$\Phi_{15}=\left(r^{3}+2 r^{2} t u+r^{2} t-2 r s^{2} u-r s^{2}-2 r s u^{2}+2 r t^{2} u+r t^{2}+r u^{2}-r-s^{2} t-2 s t u+t^{3}+t u^{2}-\right.$ t) $W_{3} W_{2}$,

$\Phi_{16}=\left(r^{2} s u+r^{2} s+r^{2} u^{2}+r^{2}+2 r s t+4 r t u-s^{3} u-s^{3}-s^{2} u^{2}+s^{2}+s t^{2} u-s t^{2}-s u^{3}-s u^{2}+s u+\right.$ $\left.s+t^{2} u^{2}+t^{2}-u^{4}+2 u^{2}-1\right) W_{3} W_{1}$,

$\Phi_{17}=-\left(r^{3} s u+r^{3} u^{2}+r^{2} s t+2 r^{2} t u-r^{2} t-r s^{3} u-r s^{2} u^{2}+2 r s^{2} u+r s t^{2} u-r s u^{3}+2 r s u^{2}+r s\right.$ $\left.u+r t^{2} u^{2}-2 r t^{2} u-r u^{4}+r u^{2}-s^{3} t-2 s^{2} t u+s^{2} t+s t^{3}+s t u^{2}+2 s t u-s t-t^{3}-t u^{2}+t\right) W_{2} W_{1}$,

$\Phi_{18}=-u\left(r^{3}+r^{2} t-r s^{2}+2 r s-r t^{2}-r u^{2}+r+s^{2} t+2 s t u-t^{3}-t u^{2}+t\right) W_{3} W_{0}$,

$\Phi_{19}=\left(r^{4} u+r^{3} t u+r^{3} t-r^{2} s^{2} u+3 r^{2} s u+r^{2} s-r^{2} t^{2} u+r^{2} t^{2}-r^{2} u^{3}+r^{2} u^{2}+r^{2} u+r^{2}+r s^{2} t u-\right.$ $r s^{2} t+2 r s t u^{2}+4 r s t-r t^{3} u-r t^{3}-r t u^{3}-r t u^{2}+5 r t u+r t-s^{3} u-s^{3}+s^{2} t^{2}-s^{2} u^{2}+s^{2}+$ $\left.3 s t^{2} u-s t^{2}-s u^{3}-s u^{2}+s u+s-t^{4}+2 t^{2}-u^{4}+2 u^{2}-1\right) W_{2} W_{0}$,

$\Phi_{20}=-u\left(r^{3} u+r^{2} t u+2 r^{2} t-r s^{2} u+2 r s u+r t^{2} u+2 r t^{2}-r u^{3}+r u-s^{2} t u-2 s^{2} t+2 s t+t^{3} u-t u^{3}+\right.$ tu) $W_{1} W_{0}$,

and

$\Psi_{1}=\left(r^{3}+r^{2} t-r s^{2}-r s u+2 r s-r t^{2}-r u^{2}+r u+s^{2} t+2 s t u-s t-t^{3}-t u+t\right) W_{n+4}^{2}$,

$\Psi_{2}=\left(-r^{3} s+r^{3} u^{2}-2 r^{2} s t u-r^{2} s t+r^{2} t u^{2}+r^{2} t u-r^{2} t+r s^{3} u+r s^{3}+r s^{2} u^{2}+r s^{2} u-r s^{2}-\right.$ $\left.r s t^{2} u-r s t^{2}-r s u^{3}+r s u^{2}+r s+r t^{2} u^{2}-r t^{2} u-r t^{2}-r u^{4}+r u^{3}+s^{2} t+2 s t u-s t-t^{3}-t u+t\right) W_{n+3}^{2}$,

$\Psi_{3}=\left(r^{3} u^{2}-r^{2} s t u-r^{2} s t+r^{2} t u^{2}+r^{2} t u-r^{2} t-r s^{2} u^{2}-r s t^{2} u-2 r s t^{2}-r s u^{3}+2 r s u^{2}+r t^{2}\right.$ $\left.u^{2}-r t^{2} u-r t^{2}-r u^{4}+r u^{3}+s^{3} t u+s^{3} t+s^{2} t u^{2}-s^{2} t u-s^{2} t-s t^{3} u+s t u^{3}+s t u-s t-t^{3}-t u+t\right)$ $W_{n+2}^{2}$,

$\Psi_{4}=u^{2}\left(r^{3}+r^{2} t-r s^{2}-r s u+2 r s-r t^{2}-r u^{2}+r u+s^{2} t+2 s t u-s t-t^{3}-t u+t\right) W_{n+1}^{2}$,

$\Psi_{5}=\left(-r^{4}-r^{3} t+r^{2} s^{2}+r^{2} s u-r^{2} s+r^{2} t^{2}-r^{2} u+r^{2}-r s^{2} t+2 r s t+r t^{3}-r t u^{2}+r t-s^{3} u-s^{3}-\right.$ $\left.s^{2} u^{2}-s^{2} u+s t^{2} u+s t^{2}+s u^{3}-s u^{2}-s u+s-t^{2} u^{2}+t^{2} u+u^{4}-u^{3}-u^{2}+u\right) W_{n+4} W_{n+3}$,

$\Psi_{6}=\left(-r^{3} s-r^{3}-r^{2} s t-r^{2} t u+r s^{3}+2 r s^{2} u-r s^{2}+r s t^{2}+3 r s u^{2}-r s+r t^{2}-r u^{2}+r-s^{3} t-3 s^{2}\right.$ $\left.t u+s t^{3}-s t u^{2}+2 s t u+s t+t^{3} u-t u^{3}+t u\right) W_{n+4} W_{n+2}$,

$\Psi_{7}=-\left(r^{3} t+r^{2} s u+r^{2} s+r^{2} t^{2}-r^{2} u+r^{2}-r s^{2} t+4 r s t-r t^{3}-3 r t u^{2}+2 r t u+r t-s^{3} u-s^{3}+s^{2}\right.$ $\left.t^{2}+s^{2} u+s^{2}+3 s t^{2} u-s t^{2}+s u^{3}-s u^{2}-s u+s-t^{4}-t^{2} u^{2}-t^{2} u+2 t^{2}-u^{3}+u^{2}+u-1\right) W_{n+4} W_{n+1}$,

$\Psi_{8}=\left(r^{3} t u-r^{3} t-r^{2} s^{2} u-r^{2} s^{2}-r^{2} s u^{2}-r^{2} s+r^{2} u^{2}-r^{2} u-r s^{2} t u-r s^{2} t+2 r s t u^{2}-2 r s t-\right.$ $r t^{3} u+r t^{3}+r t u^{3}-r t u^{2}-r t u+r t+s^{4} u+s^{4}+s^{3} u^{2}-s^{3}-s^{2} t^{2} u-s^{2} t^{2}-s^{2} u^{3}-s^{2}+s t^{2} u^{2}+$ $\left.s t^{2}-s u^{4}+2 s u^{3}-2 s u+s-t^{2} u^{2}+t^{2} u+u^{4}-u^{3}-u^{2}+u\right) W_{n+3} W_{n+2}$,

$\Psi_{9}=u\left(r^{3} s-r^{3}+r^{2} s t-r^{2} t u-r s^{3}+3 r s^{2}-r s t^{2}+r s u^{2}+2 r s u-r s+r t^{2}-r u^{2}+r+s^{3} t+s^{2} t u-\right.$ $\left.2 s^{2} t-s t^{3}-s t u^{2}+3 s t+t^{3} u-t u^{3}+t u\right) W_{n+3} W_{n+1}$,

$\Psi_{10}=u\left(r^{3} t-r^{2} s u-r^{2} s+r^{2} t^{2}+r^{2} u-r^{2}-r s^{2} t-2 r s t u-r t^{3}+r t u^{2}-r t+s^{3} u+s^{3}+s^{2} t^{2}-s^{2}\right.$ $\left.u-s^{2}+s t^{2} u-s t^{2}-s u^{3}+s u^{2}+s u-s-t^{4}+t^{2} u^{2}-t^{2} u+u^{3}-u^{2}-u+1\right) W_{n+2} W_{n+1}$, 


$$
\begin{aligned}
& \Psi_{11}=-\left(r^{3}+r^{2} t-r s^{2}-r s u+2 r s-r t^{2}-r u^{2}+r u+s^{2} t+2 s t u-s t-t^{3}-t u+t\right) W_{3}^{2}, \\
& \Psi_{12}=\left(r^{3} s-r^{3} u^{2}+2 r^{2} s t u+r^{2} s t-r^{2} t u^{2}-r^{2} t u+r^{2} t-r s^{3} u-r s^{3}-r s^{2} u^{2}-r s^{2} u+r s^{2}+r\right. \\
& \left.s t^{2} u+r s t^{2}+r s u^{3}-r s u^{2}-r s-r t^{2} u^{2}+r t^{2} u+r t^{2}+r u^{4}-r u^{3}-s^{2} t-2 s t u+s t+t^{3}+t u-t\right) W_{2}^{2}, \\
& \Psi_{13}=-\left(r^{3} u^{2}-r^{2} s t u-r^{2} s t+r^{2} t u^{2}+r^{2} t u-r^{2} t-r s^{2} u^{2}-r s t^{2} u-2 r s t^{2}-r s u^{3}+2 r s u^{2}+r t^{2}\right. \\
& \left.u^{2}-r t^{2} u-r t^{2}-r u^{4}+r u^{3}+s^{3} t u+s^{3} t+s^{2} t u^{2}-s^{2} t u-s^{2} t-s t^{3} u+s t u^{3}+s t u-s t-t^{3}-t u+t\right) \\
& W_{1}^{2}, \\
& \Psi_{14}=-u^{2}\left(r^{3}+r^{2} t-r s^{2}-r s u+2 r s-r t^{2}-r u^{2}+r u+s^{2} t+2 s t u-s t-t^{3}-t u+t\right) W_{0}^{2}, \\
& \Psi_{15}=\left(r^{4}+r^{3} t-r^{2} s^{2}-r^{2} s u+r^{2} s-r^{2} t^{2}+r^{2} u-r^{2}+r s^{2} t-2 r s t-r t^{3}+r t u^{2}-r t+s^{3} u+s^{3}+\right. \\
& \left.s^{2} u^{2}+s^{2} u-s t^{2} u-s t^{2}-s u^{3}+s u^{2}+s u-s+t^{2} u^{2}-t^{2} u-u^{4}+u^{3}+u^{2}-u\right) W_{3} W_{2}, \\
& \Psi_{16}=\left(r^{3} s+r^{3}+r^{2} s t+r^{2} t u-r s^{3}-2 r s^{2} u+r s^{2}-r s t^{2}-3 r s u^{2}+r s-r t^{2}+r u^{2}-r+s^{3} t+3 s^{2}\right. \\
& \left.t u-s t^{3}+s t u^{2}-2 s t u-s t-t^{3} u+t u^{3}-t u\right) W_{3} W_{1}, \\
& \Psi_{17}=-\left(r^{3} t u-r^{3} t-r^{2} s^{2} u-r^{2} s^{2}-r^{2} s u^{2}-r^{2} s+r^{2} u^{2}-r^{2} u-r s^{2} t u-r s^{2} t+2 r s t u^{2}-2 r s t-\right. \\
& r t^{3} u+r t^{3}+r t u^{3}-r t u^{2}-r t u+r t+s^{4} u+s^{4}+s^{3} u^{2}-s^{3}-s^{2} t^{2} u-s^{2} t^{2}-s^{2} u^{3}-s^{2}+s t^{2} u^{2}+ \\
& \left.s t^{2}-s u^{4}+2 s u^{3}-2 s u+s-t^{2} u^{2}+t^{2} u+u^{4}-u^{3}-u^{2}+u\right) W_{2} W_{1}, \\
& \Psi_{18}=\left(r^{3} t+r^{2} s u+r^{2} s+r^{2} t^{2}-r^{2} u+r^{2}-r s^{2} t+4 r s t-r t^{3}-3 r t u^{2}+2 r t u+r t-s^{3} u-s^{3}+s^{2}\right. \\
& \left.t^{2}+s^{2} u+s^{2}+3 s t^{2} u-s t^{2}+s u^{3}-s u^{2}-s u+s-t^{4}-t^{2} u^{2}-t^{2} u+2 t^{2}-u^{3}+u^{2}+u-1\right) W_{3} W_{0}, \\
& \Psi_{19}=-u\left(r^{3} s-r^{3}+r^{2} s t-r^{2} t u-r s^{3}+3 r s^{2}-r s t^{2}+r s u^{2}+2 r s u-r s+r t^{2}-r u^{2}+r+s^{3} t+s^{2} t u-\right. \\
& \left.2 s^{2} t-s t^{3}-s t u^{2}+3 s t+t^{3} u-t u^{3}+t u\right) W_{2} W_{0}, \\
& \Psi_{20}=u\left(-r^{3} t+r^{2} s u+r^{2} s-r^{2} t^{2}-r^{2} u+r^{2}+r s^{2} t+2 r s t u+r t^{3}-r t u^{2}+r t-s^{3} u-s^{3}-s^{2} t^{2}+s^{2}\right. \\
& \left.u+s^{2}-s t^{2} u+s t^{2}+s u^{3}-s u^{2}-s u+s+t^{4}-t^{2} u^{2}+t^{2} u-u^{3}+u^{2}+u-1\right) W_{1} W_{0} .
\end{aligned}
$$

Proof. First, we obtain $\sum_{k=0}^{n} W_{k}^{2}$. Using the recurrence relation

$$
W_{n+4}=r W_{n+3}+s W_{n+2}+t W_{n+1}+u W_{n}
$$

$$
u W_{n}=W_{n+4}-r W_{n+3}-s W_{n+2}-t W_{n+1}
$$


we obtain

$$
\begin{aligned}
u^{2} W_{n}^{2}= & W_{n+4}^{2}+r^{2} W_{n+3}^{2}+s^{2} W_{n+2}^{2}+t^{2} W_{n+1}^{2}-2 r W_{n+4} W_{n+3}-2 s W_{n+4} W_{n+2} \\
& -2 t W_{n+4} W_{n+1}+2 r s W_{n+3} W_{n+2}+2 r t W_{n+3} W_{n+1}+2 s t W_{n+2} W_{n+1} \\
u^{2} W_{n-1}^{2}= & W_{n+3}^{2}+r^{2} W_{n+2}^{2}+s^{2} W_{n+1}^{2}+t^{2} W_{n}^{2}-2 r W_{n+3} W_{n+2}-2 s W_{n+3} W_{n+1} \\
& -2 t W_{n+3} W_{n}+2 r s W_{n+2} W_{n+1}+2 r t W_{n+2} W_{n}+2 s t W_{n+1} W_{n} \\
& \vdots \\
u^{2} W_{1}^{2}= & W_{5}^{2}+r^{2} W_{4}^{2}+s^{2} W_{3}^{2}+t^{2} W_{2}^{2}-2 r W_{5} W_{4}-2 s W_{5} W_{3} \\
& -2 t W_{5} W_{2}+2 r s W_{4} W_{3}+2 r t W_{4} W_{2}+2 s t W_{3} W_{2} \\
u^{2} W_{0}^{2}= & W_{4}^{2}+r^{2} W_{3}^{2}+s^{2} W_{2}^{2}+t^{2} W_{1}^{2}-2 r W_{4} W_{3}-2 s W_{4} W_{2} \\
& -2 t W_{4} W_{1}+2 r s W_{3} W_{2}+2 r t W_{3} W_{1}+2 s t W_{2} W_{1}
\end{aligned}
$$

If we add the equations by side by, we get

$$
\begin{aligned}
u^{2} \sum_{k=0}^{n} W_{k}^{2}= & \left(r^{2}+s^{2}+t^{2}+1\right) \sum_{k=0}^{n} W_{k}^{2}+2(-r+r s+s t) \sum_{k=0}^{n} W_{k+1} W_{k} \\
& +2(-s+r t) \sum_{k=0}^{n} W_{k+2} W_{k}-2 t \sum_{k=0}^{n} W_{k+3} W_{k}+W_{n+4}^{2}+\left(r^{2}+1\right) W_{n+3}^{2} \\
& +\left(r^{2}+s^{2}+1\right) W_{n+2}^{2}+\left(r^{2}+s^{2}+t^{2}+1\right) W_{n+1}^{2}-2 r W_{n+4} W_{n+3}-2 s W_{n+4} W_{n+2} \\
& -2 t W_{n+4} W_{n+1}+2 r(s-1) W_{n+3} W_{n+2}+2(-s+r t) W_{n+3} W_{n+1} \\
& +2(-r+r s+s t) W_{n+2} W_{n+1}-W_{3}^{2}-\left(r^{2}+1\right) W_{2}^{2}-\left(r^{2}+s^{2}+1\right) W_{1}^{2} \\
& -\left(r^{2}+s^{2}+t^{2}+1\right) W_{0}^{2}+2 r W_{3} W_{2}+2 s W_{3} W_{1}-2 r(s-1) W_{2} W_{1} \\
+ & 2 t W_{3} W_{0}-2(-s+r t) W_{2} W_{0}-2(-r+r s+s t) W_{1} W_{0} .
\end{aligned}
$$

Next we obtain $\sum_{k=0}^{n} W_{k+1} W_{k}$. Multiplying the both side of the recurrence relation

$$
u W_{n}=W_{n+4}-r W_{n+3}-s W_{n+2}-t W_{n+1}
$$

by $W_{n+1}$ we get

$$
u W_{n+1} W_{n}=W_{n+4} W_{n+1}-r W_{n+3} W_{n+1}-s W_{n+2} W_{n+1}-t W_{n+1}^{2}
$$


Then using last recurrence relation, we obtain

$$
\begin{aligned}
u W_{n+1} W_{n}= & W_{n+4} W_{n+1}-r W_{n+3} W_{n+1}-s W_{n+2} W_{n+1}-t W_{n+1}^{2} \\
u W_{n} W_{n-1}= & W_{n+3} W_{n}-r W_{n+2} W_{n}-s W_{n+1} W_{n}-t W_{n}^{2} \\
u W_{n-1} W_{n-2}= & W_{n+2} W_{n-1}-r W_{n+1} W_{n-1}-s W_{n} W_{n-1}-t W_{n-1}^{2} \\
& \vdots \\
u W_{3} W_{2}= & W_{6} W_{3}-r W_{5} W_{3}-s W_{4} W_{3}-t W_{3}^{2} \\
u W_{2} W_{1}= & W_{5} W_{2}-r W_{4} W_{2}-s W_{3} W_{2}-t W_{2}^{2} \\
u W_{1} W_{0}= & W_{4} W_{1}-r W_{3} W_{1}-s W_{2} W_{1}-t W_{1}^{2}
\end{aligned}
$$

If we add the equations by side by, we get

$$
\begin{aligned}
u \sum_{k=0}^{n} W_{k+1} W_{k}= & \left(W_{n+4} W_{n+1}-W_{3} W_{0}+\sum_{k=0}^{n} W_{k+3} W_{k}\right) \\
& -r\left(W_{n+3} W_{n+1}-W_{2} W_{0}+\sum_{k=0}^{n} W_{k+2} W_{k}\right) \\
& -s\left(W_{n+2} W_{n+1}-W_{1} W_{0}+\sum_{k=0}^{n} W_{k+1} W_{k}\right) \\
& -t\left(W_{n+1}^{2}-W_{0}^{2}+\sum_{k=0}^{n} W_{k}^{2}\right) .
\end{aligned}
$$

Next we obtain $\sum_{k=0}^{n} W_{k+2} W_{k}$. Multiplying the both side of the recurrence relation

$$
u W_{n}=W_{n+4}-r W_{n+3}-s W_{n+2}-t W_{n+1}
$$

by $W_{n+2}$ we get

$$
u W_{n+2} W_{n}=W_{n+4} W_{n+2}-r W_{n+3} W_{n+2}-s W_{n+2}^{2}-t W_{n+2} W_{n+1}
$$

Then using last recurrence relation, we obtain

$$
\begin{aligned}
u W_{n+2} W_{n}= & W_{n+4} W_{n+2}-r W_{n+3} W_{n+2}-s W_{n+2}^{2}-t W_{n+2} W_{n+1} \\
u W_{n+1} W_{n-1}= & W_{n+3} W_{n+1}-r W_{n+2} W_{n+1}-s W_{n+1}^{2}-t W_{n+1} W_{n} \\
u W_{n} W_{n-2}= & W_{n+2} W_{n}-r W_{n+1} W_{n}-s W_{n}^{2}-t W_{n} W_{n-1} \\
& \vdots \\
u W_{3} W_{1}= & W_{5} W_{3}-r W_{4} W_{3}-s W_{3}^{2}-t W_{3} W_{2} \\
u W_{2} W_{0}= & W_{4} W_{2}-r W_{3} W_{2}-s W_{2}^{2}-t W_{2} W_{1}
\end{aligned}
$$


If we add the equations by side by, we get

$$
\begin{aligned}
u \sum_{k=0}^{n} W_{k+2} W_{k}= & \left(W_{n+4} W_{n+2}+W_{n+3} W_{n+1}-W_{3} W_{1}-W_{2} W_{0}+\sum_{k=0}^{n} W_{k+2} W_{k}\right) \\
& -r\left(W_{n+3} W_{n+2}+W_{n+2} W_{n+1}-W_{2} W_{1}-W_{1} W_{0}+\sum_{k=0}^{n} W_{k+1} W_{k}\right) \\
& -s\left(W_{n+2}^{2}+W_{n+1}^{2}-W_{1}^{2}-W_{0}^{2}+\sum_{k=0}^{n} W_{k}^{2}\right) \\
& -t\left(W_{n+2} W_{n+1}-W_{1} W_{0}+\sum_{k=0}^{n} W_{k+1} W_{k}\right) .
\end{aligned}
$$

Next we obtain $\sum_{k=0}^{n} W_{k+3} W_{k}$. Multiplying the both side of the recurrence relation

$$
u W_{n}=W_{n+4}-r W_{n+3}-s W_{n+2}-t W_{n+1}
$$

by $W_{n+3}$ we get

$$
u W_{n+3} W_{n}=W_{n+4} W_{n+3}-r W_{n+3}^{2}-s W_{n+3} W_{n+2}-t W_{n+3} W_{n+1}
$$

Then using last recurrence relation, we obtain

$$
\begin{aligned}
u W_{n+3} W_{n}= & W_{n+4} W_{n+3}-r W_{n+3}^{2}-s W_{n+3} W_{n+2}-t W_{n+3} W_{n+1} \\
u W_{n+2} W_{n-1}= & W_{n+3} W_{n+2}-r W_{n+2}^{2}-s W_{n+2} W_{n+1}-t W_{n+2} W_{n} \\
u W_{n+1} W_{n-2}= & W_{n+2} W_{n+1}-r W_{n+1}^{2}-s W_{n+1} W_{n}-t W_{n+1} W_{n-1} \\
& \vdots \\
u W_{5} W_{2}= & W_{6} W_{5}-r W_{5}^{2}-s W_{5} W_{4}-t W_{5} W_{3} \\
u W_{4} W_{1}= & W_{5} W_{4}-r W_{4}^{2}-s W_{4} W_{3}-t W_{4} W_{2} \\
u W_{3} W_{0}= & W_{4} W_{3}-r W_{3}^{2}-s W_{3} W_{2}-t W_{3} W_{1}
\end{aligned}
$$

If we add the equations by side by, we get

$$
\begin{aligned}
\left(2 \sum_{k=0}^{n} W_{k+3} W_{k}=\right. & \left(W_{n+4} W_{n+3}+W_{n+3} W_{n+2}+W_{n+2} W_{n+1}-W_{3} W_{2}-W_{2} W_{1}-W_{1} W_{0}+\sum_{k=0}^{n} W_{k+1} W_{k}\right) \\
& -r\left(W_{n+3}^{2}+W_{n+2}^{2}+W_{n+1}^{2}-W_{2}^{2}-W_{1}^{2}-W_{0}^{2}+\sum_{k=0}^{n} W_{k}^{2}\right) \\
& -s\left(W_{n+3} W_{n+2}+W_{n+2} W_{n+1}-W_{2} W_{1}-W_{1} W_{0}+\sum_{k=0}^{n} W_{k+1} W_{k}\right) \\
& -t\left(W_{n+3} W_{n+1}-W_{2} W_{0}+\sum_{k=0}^{n} W_{k+2} W_{k}\right) .
\end{aligned}
$$

Solving the system (2.1)-(2.2)-(2.3)-(2.4), the results in (a), (b), (c) and (d) follow. 


\section{Specific Cases}

In this section, we present the closed form solutions (identities) of the sums $\sum_{k=0}^{n} W_{k}^{2}, \sum_{k=0}^{n} W_{k+1} W_{k}$ , $\sum_{k=0}^{n} W_{k+2} W_{k}$ and $\sum_{k=0}^{n} W_{k+3} W_{k}$ for the specific case of sequence $\left\{W_{n}\right\}$.

Taking $r=s=t=u=1$ in Theorem 2.1, we obtain the following proposition.

Proposition 3.1. If $r=s=t=u=1$ then for $n \geq 0$ we have the following formulas:

(a): $\sum_{k=0}^{n} W_{k}^{2}=\frac{1}{3}\left(-W_{n+4}^{2}-3 W_{n+3}^{2}-4 W_{n+2}^{2}-4 W_{n+1}^{2}+3 W_{n+4} W_{n+3}+2 W_{n+4} W_{n+2}+W_{n+4} W_{n+1}-\right.$ $\left.W_{n+2} W_{n+1}+W_{3}^{2}+3 W_{2}^{2}+4 W_{1}^{2}+4 W_{0}^{2}-3 W_{3} W_{2}-2 W_{3} W_{1}-W_{3} W_{0}+W_{1} W_{0}\right)$.

(b): $\sum_{k=0}^{n} W_{k+1} W_{k}=\frac{1}{6}\left(W_{n+4}^{2}+3 W_{n+3}^{2}+W_{n+2}^{2}+W_{n+1}^{2}-3 W_{n+4} W_{n+3}+W_{n+4} W_{n+2}-W_{n+4} W_{n+1}-3\right.$ $W_{n+3} W_{n+2}+3 W_{n+3} W_{n+1}-5 W_{n+2} W_{n+1}-W_{3}^{2}-3 W_{2}^{2}-W_{1}^{2}-W_{0}^{2}+3 W_{3} W_{2}-W_{3} W_{1}+W_{3} W_{0}+$ $\left.3 W_{2} W_{1}-3 W_{2} W_{0}+5 W_{1} W_{0}\right)$.

(c): $\sum_{k=0}^{n} W_{k+2} W_{k}=\frac{1}{6}\left(W_{n+4}^{2}+4 W_{n+2}^{2}+W_{n+1}^{2}-5 W_{n+4} W_{n+2}+2 W_{n+4} W_{n+1}+3 W_{n+3} W_{n+2}-\right.$ $\left.9 W_{n+3} W_{n+1}+4 W_{n+2} W_{n+1}-W_{3}^{2}-4 W_{1}^{2}-W_{0}^{2}+5 W_{3} W_{1}-2 W_{3} W_{0}-3 W_{2} W_{1}+9 W_{2} W_{0}-4 W_{1} W_{0}\right)$.

(d): $\sum_{k=0}^{n} W_{k+3} W_{k}=\frac{1}{6}\left(W_{n+4}^{2}-2 W_{n+2}^{2}+W_{n+1}^{2}+W_{n+4} W_{n+2}-4 W_{n+4} W_{n+1}-3 W_{n+3} W_{n+2}+\right.$ $\left.3 W_{n+3} W_{n+1}-2 W_{n+2} W_{n+1}-W_{3}^{2}+2 W_{1}^{2}-W_{0}^{2}-W_{3} W_{1}+4 W_{3} W_{0}+3 W_{2} W_{1}-3 W_{2} W_{0}+2 W_{1} W_{0}\right)$.

From the above proposition, we have the following corollary which gives sum formulas of Tetranacci numbers (take $W_{n}=M_{n}$ with $M_{0}=0, M_{1}=1, M_{2}=1, M_{3}=2$ ).

Corollary 3.2. For $n \geq 0$, Tetranacci numbers have the following properties:

(a): $\sum_{k=0}^{n} M_{k}^{2}=\frac{1}{3}\left(-M_{n+4}^{2}-3 M_{n+3}^{2}-4 M_{n+2}^{2}-4 M_{n+1}^{2}+3 M_{n+4} M_{n+3}+2 M_{n+4} M_{n+2}+M_{n+4} M_{n+1}-\right.$ $\left.M_{n+2} M_{n+1}+1\right)$.

(b): $\sum_{k=0}^{n} M_{k+1} M_{k}=\frac{1}{6}\left(M_{n+4}^{2}+3 M_{n+3}^{2}+M_{n+2}^{2}+M_{n+1}^{2}-3 M_{n+4} M_{n+3}+M_{n+4} M_{n+2}-M_{n+4} M_{n+1}-3\right.$ $\left.M_{n+3} M_{n+2}+3 M_{n+3} M_{n+1}-5 M_{n+2} M_{n+1}-1\right)$.

(c): $\sum_{k=0}^{n} M_{k+2} M_{k}=\frac{1}{6}\left(M_{n+4}^{2}+4 M_{n+2}^{2}+M_{n+1}^{2}-5 M_{n+4} M_{n+2}+2 M_{n+4} M_{n+1}+3 M_{n+3} M_{n+2}-\right.$ $\left.9 M_{n+3} M_{n+1}+4 M_{n+2} M_{n+1}-1\right)$.

(d): $\sum_{k=0}^{n} M_{k+3} M_{k}=\frac{1}{6}\left(M_{n+4}^{2}-2 M_{n+2}^{2}+M_{n+1}^{2}+M_{n+4} M_{n+2}-4 M_{n+4} M_{n+1}-3 M_{n+3} M_{n+2}+\right.$ $\left.3 M_{n+3} M_{n+1}-2 M_{n+2} M_{n+1}-1\right)$.

Taking $W_{n}=R_{n}$ with $R_{0}=4, R_{1}=1, R_{2}=3, R_{3}=7$ in the above proposition, we have the following corollary which presents sum formulas of Tetranacci-Lucas numbers.

Corollary 3.3. For $n \geq 0$, Tetranacci-Lucas numbers have the following properties:

(a): $\sum_{k=0}^{n} R_{k}^{2}=\frac{1}{3}\left(-R_{n+4}^{2}-3 R_{n+3}^{2}-4 R_{n+2}^{2}-4 R_{n+1}^{2}+3 R_{n+4} R_{n+3}+2 R_{n+4} R_{n+2}+R_{n+4} R_{n+1}-\right.$ $\left.R_{n+2} R_{n+1}+43\right)$.

(b): $\sum_{k=0}^{n} R_{k+1} R_{k}=\frac{1}{6}\left(R_{n+4}^{2}+3 R_{n+3}^{2}+R_{n+2}^{2}+R_{n+1}^{2}-3 R_{n+4} R_{n+3}+R_{n+4} R_{n+2}-R_{n+4} R_{n+1}-3\right.$ $\left.R_{n+3} R_{n+2}+3 R_{n+3} R_{n+1}-5 R_{n+2} R_{n+1}-16\right)$. 
(c): $\sum_{k=0}^{n} R_{k+2} R_{k}=\frac{1}{6}\left(R_{n+4}^{2}+4 R_{n+2}^{2}+R_{n+1}^{2}-5 R_{n+4} R_{n+2}+2 R_{n+4} R_{n+1}+3 R_{n+3} R_{n+2}-9 R_{n+3} R_{n+1}+\right.$ $\left.4 R_{n+2} R_{n+1}-7\right)$.

(d): $\sum_{k=0}^{n} R_{k+3} R_{k}=\frac{1}{6}\left(R_{n+4}^{2}-2 R_{n+2}^{2}+R_{n+1}^{2}+R_{n+4} R_{n+2}-4 R_{n+4} R_{n+1}-3 R_{n+3} R_{n+2}+3 R_{n+3} R_{n+1}-\right.$ $\left.2 R_{n+2} R_{n+1}+23\right)$.

Taking $r=2, s=1, t=1, u=1$ in Theorem 2.1, we obtain the following proposition.

Proposition 3.4. If $r=2, s=1, t=1, u=1$ then for $n \geq 0$ we have the following formulas:

(a): $\sum_{k=0}^{n} W_{k}^{2}=\frac{1}{56}\left(-9 W_{n+4}^{2}-57 W_{n+3}^{2}-68 W_{n+2}^{2}-65 W_{n+1}^{2}+42 W_{n+4} W_{n+3}+20 W_{n+4} W_{n+2}+6\right.$ $W_{n+4} W_{n+1}-4 W_{n+3} W_{n+2}+2 W_{n+3} W_{n+1}-12 W_{n+2} W_{n+1}+9 W_{3}^{2}+57 W_{2}^{2}+68 W_{1}^{2}+65 W_{0}^{2}-42 W_{3} W_{2}-$ $\left.20 W_{3} W_{1}-6 W_{3} W_{0}+4 W_{2} W_{1}-2 W_{2} W_{0}+12 W_{1} W_{0}\right)$.

(b): $\sum_{k=0}^{n} W_{k+1} W_{k}=\frac{1}{56}\left(3 W_{n+4}^{2}+19 W_{n+3}^{2}+4 W_{n+2}^{2}+3 W_{n+1}^{2}-14 W_{n+4} W_{n+3}+12 W_{n+4} W_{n+2}-2\right.$ $W_{n+4} W_{n+1}-36 W_{n+3} W_{n+2}+18 W_{n+3} W_{n+1}-52 W_{n+2} W_{n+1}-3 W_{3}^{2}-19 W_{2}^{2}-4 W_{1}^{2}-3 W_{0}^{2}+14 W_{3} W_{2}-$ $\left.12 W_{3} W_{1}+2 W_{3} W_{0}+36 W_{2} W_{1}-18 W_{2} W_{0}+52 W_{1} W_{0}\right)$.

(c): $\sum_{k=0}^{n} W_{k+2} W_{k}=\frac{1}{8}\left(W_{n+4}^{2}+W_{n+3}^{2}+4 W_{n+2}^{2}+W_{n+1}^{2}-2 W_{n+4} W_{n+3}-4 W_{n+4} W_{n+2}+2 W_{n+4} W_{n+1}+\right.$ $4 W_{n+3} W_{n+2}-10 W_{n+3} W_{n+1}+4 W_{n+2} W_{n+1}-W_{3}^{2}-W_{2}^{2}-4 W_{1}^{2}-W_{0}^{2}+2 W_{3} W_{2}+4 W_{3} W_{1}-2 W_{3} W_{0}-$ $\left.4 W_{2} W_{1}+10 W_{2} W_{0}-4 W_{1} W_{0}\right)$.

(d): $\sum_{k=0}^{n} W_{k+3} W_{k}=\frac{1}{56}\left(11 W_{n+4}^{2}-5 W_{n+3}^{2}-4 W_{n+2}^{2}+11 W_{n+1}^{2}-14 W_{n+4} W_{n+3}-12 W_{n+4} W_{n+2}-26\right.$ $W_{n+4} W_{n+1}-20 W_{n+3} W_{n+2}+10 W_{n+3} W_{n+1}-4 W_{n+2} W_{n+1}-11 W_{3}^{2}+5 W_{2}^{2}+4 W_{1}^{2}-11 W_{0}^{2}+14 W_{3} W_{2}+$ $\left.12 W_{3} W_{1}+26 W_{3} W_{0}+20 W_{2} W_{1}-10 W_{2} W_{0}+4 W_{1} W_{0}\right)$.

From the last proposition, we have the following corollary which gives sum formulas of fourth-order Pell numbers (take $W_{n}=P_{n}$ with $P_{0}=0, P_{1}=1, P_{2}=2, P_{3}=5$ ).

Corollary 3.5. For $n \geq 0$, fourth-order Pell numbers have the following properties:

(a): $\sum_{k=0}^{n} P_{k}^{2}=\frac{1}{56}\left(-9 P_{n+4}^{2}-57 P_{n+3}^{2}-68 P_{n+2}^{2}-65 P_{n+1}^{2}+42 P_{n+4} P_{n+3}+20 P_{n+4} P_{n+2}+6 P_{n+4} P_{n+1}-\right.$ $\left.4 P_{n+3} P_{n+2}+2 P_{n+3} P_{n+1}-12 P_{n+2} P_{n+1}+9\right)$.

(b): $\sum_{k=0}^{n} P_{k+1} P_{k}=\frac{1}{56}\left(3 P_{n+4}^{2}+19 P_{n+3}^{2}+4 P_{n+2}^{2}+3 P_{n+1}^{2}-14 P_{n+4} P_{n+3}+12 P_{n+4} P_{n+2}-2 P_{n+4} P_{n+1}-\right.$ $\left.36 P_{n+3} P_{n+2}+18 P_{n+3} P_{n+1}-52 P_{n+2} P_{n+1}-3\right)$.

(c): $\sum_{k=0}^{n} P_{k+2} P_{k}=\frac{1}{8}\left(P_{n+4}^{2}+P_{n+3}^{2}+4 P_{n+2}^{2}+P_{n+1}^{2}-2 P_{n+4} P_{n+3}-4 P_{n+4} P_{n+2}+2 P_{n+4} P_{n+1}+\right.$ $\left.4 P_{n+3} P_{n+2}-10 P_{n+3} P_{n+1}+4 P_{n+2} P_{n+1}-1\right)$.

(d): $\sum_{k=0}^{n} P_{k+3} P_{k}=\frac{1}{56}\left(11 P_{n+4}^{2}-5 P_{n+3}^{2}-4 P_{n+2}^{2}+11 P_{n+1}^{2}-14 P_{n+4} P_{n+3}-12 P_{n+4} P_{n+2}-26\right.$ $\left.P_{n+4} P_{n+1}-20 P_{n+3} P_{n+2}+10 P_{n+3} P_{n+1}-4 P_{n+2} P_{n+1}-11\right)$.

Taking $W_{n}=Q_{n}$ with $Q_{0}=4, Q_{1}=2, Q_{2}=6, Q_{3}=17$ in the last proposition, we have the following corollary which presents sum formulas of fourth-order Pell-Lucas numbers.

Corollary 3.6. For $n \geq 0$, fourth-order Pell-Lucas numbers have the following properties: 
(a): $\sum_{k=0}^{n} Q_{k}^{2}=\frac{1}{56}\left(-9 Q_{n+4}^{2}-57 Q_{n+3}^{2}-68 Q_{n+2}^{2}-65 Q_{n+1}^{2}+42 Q_{n+4} Q_{n+3}+20 Q_{n+4} Q_{n+2}+6\right.$ $\left.Q_{n+4} Q_{n+1}-4 Q_{n+3} Q_{n+2}+2 Q_{n+3} Q_{n+1}-12 Q_{n+2} Q_{n+1}+689\right)$.

(b): $\sum_{k=0}^{n} Q_{k+1} Q_{k}=\frac{1}{56}\left(3 Q_{n+4}^{2}+19 Q_{n+3}^{2}+4 Q_{n+2}^{2}+3 Q_{n+1}^{2}-14 Q_{n+4} Q_{n+3}+12 Q_{n+4} Q_{n+2}-2\right.$ $\left.Q_{n+4} Q_{n+1}-36 Q_{n+3} Q_{n+2}+18 Q_{n+3} Q_{n+1}-52 Q_{n+2} Q_{n+1}-43\right)$.

(c): $\sum_{k=0}^{n} Q_{k+2} Q_{k}=\frac{1}{8}\left(Q_{n+4}^{2}+Q_{n+3}^{2}+4 Q_{n+2}^{2}+Q_{n+1}^{2}-2 Q_{n+4} Q_{n+3}-4 Q_{n+4} Q_{n+2}+2 Q_{n+4} Q_{n+1}+\right.$ $\left.4 Q_{n+3} Q_{n+2}-10 Q_{n+3} Q_{n+1}+4 Q_{n+2} Q_{n+1}+7\right)$.

(d): $\sum_{k=0}^{n} Q_{k+3} Q_{k}=\frac{1}{56}\left(11 Q_{n+4}^{2}-5 Q_{n+3}^{2}-4 Q_{n+2}^{2}+11 Q_{n+1}^{2}-14 Q_{n+4} Q_{n+3}-12 Q_{n+4} Q_{n+2}-26\right.$ $\left.Q_{n+4} Q_{n+1}-20 Q_{n+3} Q_{n+2}+10 Q_{n+3} Q_{n+1}-4 Q_{n+2} Q_{n+1}+477\right)$.

From the last proposition, we have the following corollary which gives sum formulas of modified fourthorder Pell numbers (take $W_{n}=E_{n}$ with $E_{0}=0, E_{1}=1, E_{2}=1, E_{3}=3$ ).

Corollary 3.7. For $n \geq 0$, modified fourth-order Pell numbers have the following properties:

(a): $\sum_{k=0}^{n} E_{k}^{2}=\frac{1}{56}\left(-9 E_{n+4}^{2}-57 E_{n+3}^{2}-68 E_{n+2}^{2}-65 E_{n+1}^{2}+42 E_{n+4} E_{n+3}+20 E_{n+4} E_{n+2}+6 E_{n+4} E_{n+1}-\right.$ $\left.4 E_{n+3} E_{n+2}+2 E_{n+3} E_{n+1}-12 E_{n+2} E_{n+1}+24\right)$.

(b): $\sum_{k=0}^{n} E_{k+1} E_{k}=\frac{1}{56}\left(3 E_{n+4}^{2}+19 E_{n+3}^{2}+4 E_{n+2}^{2}+3 E_{n+1}^{2}-14 E_{n+4} E_{n+3}+12 E_{n+4} E_{n+2}-2\right.$ $\left.E_{n+4} E_{n+1}-36 E_{n+3} E_{n+2}+18 E_{n+3} E_{n+1}-52 E_{n+2} E_{n+1}-8\right)$.

(c): $\sum_{k=0}^{n} E_{k+2} E_{k}=\frac{1}{8}\left(E_{n+4}^{2}+E_{n+3}^{2}+4 E_{n+2}^{2}+E_{n+1}^{2}-2 E_{n+4} E_{n+3}-4 E_{n+4} E_{n+2}+2 E_{n+4} E_{n+1}+\right.$ $\left.4 E_{n+3} E_{n+2}-10 E_{n+3} E_{n+1}+4 E_{n+2} E_{n+1}\right)$.

(d): $\sum_{k=0}^{n} E_{k+3} E_{k}=\frac{1}{56}\left(11 E_{n+4}^{2}-5 E_{n+3}^{2}-4 E_{n+2}^{2}+11 E_{n+1}^{2}-14 E_{n+4} E_{n+3}-12 E_{n+4} E_{n+2}-26\right.$ $\left.E_{n+4} E_{n+1}-20 E_{n+3} E_{n+2}+10 E_{n+3} E_{n+1}-4 E_{n+2} E_{n+1}+8\right)$.

Taking $r=2, s=3, t=5$ in Theorem 2.1, we obtain the following Proposition.

Proposition 3.8. If $r=2, s=3, t=5$ then for $n \geq 0$ we have the following formulas:

(a): $\sum_{k=0}^{n} W_{k}^{2}=\frac{1}{7968}\left(299 W_{n+4}^{2}+2155 W_{n+3}^{2}+2608 W_{n+2}^{2}+6683 W_{n+1}^{2}-1526 W_{n+4} W_{n+3}-1048\right.$ $W_{n+4} W_{n+2}-2310 W_{n+4} W_{n+1}+1560 W_{n+3} W_{n+2}+5222 W_{n+3} W_{n+1}+4760 W_{n+2} W_{n+1}-299 W_{3}^{2}-$ $2155 W_{2}^{2}-2608 W_{1}^{2}-6683 W_{0}^{2}+1526 W_{3} W_{2}+1048 W_{3} W_{1}+2310 W_{3} W_{0}-1560 W_{2} W_{1}-5222 W_{2} W_{0}-$ $\left.4760 W_{1} W_{0}\right)$.

(b): $\sum_{k=0}^{n} W_{k+1} W_{k}=\frac{1}{2656}\left(-55 W_{n+4}^{2}-503 W_{n+3}^{2}-80 W_{n+2}^{2}-2695 W_{n+1}^{2}+334 W_{n+4} W_{n+3}+24 W_{n+4} W_{n+2}+\right.$ $798 W_{n+4} W_{n+1}-56 W_{n+3} W_{n+2}-2142 W_{n+3} W_{n+1}-920 W_{n+2} W_{n+1}+55 W_{3}^{2}+503 W_{2}^{2}+80 W_{1}^{2}+$ $\left.2695 W_{0}^{2}-334 W_{3} W_{2}-24 W_{3} W_{1}-798 W_{3} W_{0}+56 W_{2} W_{1}+2142 W_{2} W_{0}+920 W_{1} W_{0}\right)$.

(c): $\sum_{k=0}^{n} W_{k+2} W_{k}=\frac{1}{7968}\left(43 W_{n+4}^{2}+683 W_{n+3}^{2}-5008 W_{n+2}^{2}+2107 W_{n+1}^{2}-406 W_{n+4} W_{n+3}+1768\right.$ $W_{n+4} W_{n+2}-1638 W_{n+4} W_{n+1}-3240 W_{n+3} W_{n+2}+6214 W_{n+3} W_{n+1}-8456 W_{n+2} W_{n+1}-43 W_{3}^{2}-$ $683 W_{2}^{2}+5008 W_{1}^{2}-2107 W_{0}^{2}+406 W_{3} W_{2}-1768 W_{3} W_{1}+1638 W_{3} W_{0}+3240 W_{2} W_{1}-6214 W_{2} W_{0}+$ $\left.8456 W_{1} W_{0}\right)$. 
(d): $\sum_{k=0}^{n} W_{k+3} W_{k}=\frac{1}{2656}\left(-23 W_{n+4}^{2}-983 W_{n+3}^{2}+208 W_{n+2}^{2}-1127 W_{n+1}^{2}+526 W_{n+4} W_{n+3}-328 W_{n+4} W_{n+2}+\right.$ $382 W_{n+4} W_{n+1}-120 W_{n+3} W_{n+2}-3262 W_{n+3} W_{n+1}+1064 W_{n+2} W_{n+1}+23 W_{3}^{2}+983 W_{2}^{2}-208 W_{1}^{2}+$ $\left.1127 W_{0}^{2}-526 W_{3} W_{2}+328 W_{3} W_{1}-382 W_{3} W_{0}+120 W_{2} W_{1}+3262 W_{2} W_{0}-1064 W_{1} W_{0}\right)$.

From the last proposition, we have the following corollary which gives sum formulas of 4-primes numbers $\left(\right.$ take $W_{n}=G_{n}$ with $\left.G_{0}=0, G_{1}=0, G_{2}=1, G_{3}=2\right)$.

Corollary 3.9. For $n \geq 0$, 4-primes numbers have the following properties:

(a): $\sum_{k=0}^{n} G_{k}^{2}=\frac{1}{7968}\left(299 G_{n+4}^{2}+2155 G_{n+3}^{2}+2608 G_{n+2}^{2}+6683 G_{n+1}^{2}-1526 G_{n+4} G_{n+3}-1048 G_{n+4} G_{n+2}-\right.$ $\left.2310 G_{n+4} G_{n+1}+1560 G_{n+3} G_{n+2}+5222 G_{n+3} G_{n+1}+4760 G_{n+2} G_{n+1}-299\right)$.

(b): $\sum_{k=0}^{n} G_{k+1} G_{k}=\frac{1}{2656}\left(-55 G_{n+4}^{2}-503 G_{n+3}^{2}-80 G_{n+2}^{2}-2695 G_{n+1}^{2}+334 G_{n+4} G_{n+3}+24 G_{n+4} G_{n+2}+\right.$ $\left.798 G_{n+4} G_{n+1}-56 G_{n+3} G_{n+2}-2142 G_{n+3} G_{n+1}-920 G_{n+2} G_{n+1}+55\right)$.

(d): $\sum_{k=0}^{n} G_{k+2} G_{k}=\frac{1}{7968}\left(43 G_{n+4}^{2}+683 G_{n+3}^{2}-5008 G_{n+2}^{2}+2107 G_{n+1}^{2}-406 G_{n+4} G_{n+3}+1768\right.$ $\left.G_{n+4} G_{n+2}-1638 G_{n+4} G_{n+1}-3240 G_{n+3} G_{n+2}+6214 G_{n+3} G_{n+1}-8456 G_{n+2} G_{n+1}-43\right)$.

(c): $\sum_{k=0}^{n} G_{k+3} G_{k}=\frac{1}{2656}\left(-23 G_{n+4}^{2}-983 G_{n+3}^{2}+208 G_{n+2}^{2}-1127 G_{n+1}^{2}+526 G_{n+4} G_{n+3}-328 G_{n+4} G_{n+2}+\right.$ $\left.382 G_{n+4} G_{n+1}-120 G_{n+3} G_{n+2}-3262 G_{n+3} G_{n+1}+1064 G_{n+2} G_{n+1}+23\right)$.

Taking $W_{n}=H_{n}$ with $H_{0}=4, H_{1}=2, H_{2}=10, H_{3}=41$ in the last proposition, we have the following corollary which presents sum formulas of Lucas 4-primes numbers.

Corollary 3.10. For $n \geq 0$, Lucas 4-primes numbers have the following properties:

(a): $\sum_{k=0}^{n} H_{k}^{2}=\frac{1}{7968}\left(299 H_{n+4}^{2}+2155 H_{n+3}^{2}+2608 H_{n+2}^{2}+6683 H_{n+1}^{2}-1526 H_{n+4} H_{n+3}-1048 H_{n+4} H_{n+2}-\right.$ $\left.2310 H_{n+4} H_{n+1}+1560 H_{n+3} H_{n+2}+5222 H_{n+3} H_{n+1}+4760 H_{n+2} H_{n+1}-23203\right)$.

(b): $\sum_{k=0}^{n} H_{k+1} H_{k}=\frac{1}{2656}\left(-55 H_{n+4}^{2}-503 H_{n+3}^{2}-80 H_{n+2}^{2}-2695 H_{n+1}^{2}+334 H_{n+4} H_{n+3}+24 H_{n+4} H_{n+2}+\right.$ $\left.798 H_{n+4} H_{n+1}-56 H_{n+3} H_{n+2}-2142 H_{n+3} H_{n+1}-920 H_{n+2} H_{n+1}+10575\right)$.

(c): $\sum_{k=0}^{n} H_{k+2} H_{k}=\frac{1}{7968}\left(43 H_{n+4}^{2}+683 H_{n+3}^{2}-5008 H_{n+2}^{2}+2107 H_{n+1}^{2}-406 H_{n+4} H_{n+3}+1768\right.$ $\left.H_{n+4} H_{n+2}-1638 H_{n+4} H_{n+1}-3240 H_{n+3} H_{n+2}+6214 H_{n+3} H_{n+1}-8456 H_{n+2} H_{n+1}+19741\right)$.

(d): $\sum_{k=0}^{n} H_{k+3} H_{k}=\frac{1}{2656}\left(-23 H_{n+4}^{2}-983 H_{n+3}^{2}+208 H_{n+2}^{2}-1127 H_{n+1}^{2}+526 H_{n+4} H_{n+3}-328 H_{n+4} H_{n+2}+\right.$ $\left.382 H_{n+4} H_{n+1}-120 H_{n+3} H_{n+2}-3262 H_{n+3} H_{n+1}+1064 H_{n+2} H_{n+1}+27119\right)$.

From the last proposition, we have the following corollary which gives sum formulas of modified 4-primes numbers (take $W_{n}=E_{n}$ with $E_{0}=0, E_{1}=0, E_{2}=1, E_{3}=1$ ).

Corollary 3.11. For $n \geq 0$, modified 4-primes numbers have the following properties:

(a): $\sum_{k=0}^{n} E_{k}^{2}=\frac{1}{7968}\left(299 E_{n+4}^{2}+2155 E_{n+3}^{2}+2608 E_{n+2}^{2}+6683 E_{n+1}^{2}-1526 E_{n+4} E_{n+3}-1048 E_{n+4} E_{n+2}-\right.$ $\left.2310 E_{n+4} E_{n+1}+1560 E_{n+3} E_{n+2}+5222 E_{n+3} E_{n+1}+4760 E_{n+2} E_{n+1}-928\right)$.

(b): $\sum_{k=0}^{n} E_{k+1} E_{k}=\frac{1}{2656}\left(-55 E_{n+4}^{2}-503 E_{n+3}^{2}-80 E_{n+2}^{2}-2695 E_{n+1}^{2}+334 E_{n+4} E_{n+3}+24 E_{n+4} E_{n+2}+\right.$ $\left.798 E_{n+4} E_{n+1}-56 E_{n+3} E_{n+2}-2142 E_{n+3} E_{n+1}-920 E_{n+2} E_{n+1}+224\right)$. 
(c): $\sum_{k=0}^{n} E_{k+2} E_{k}=\frac{1}{7968}\left(43 E_{n+4}^{2}+683 E_{n+3}^{2}-5008 E_{n+2}^{2}+2107 E_{n+1}^{2}-406 E_{n+4} E_{n+3}+1768 E_{n+4} E_{n+2}-\right.$ $\left.1638 E_{n+4} E_{n+1}-3240 E_{n+3} E_{n+2}+6214 E_{n+3} E_{n+1}-8456 E_{n+2} E_{n+1}-320\right)$.

(d): $\sum_{k=0}^{n} E_{k+3} E_{k}=\frac{1}{2656}\left(-23 E_{n+4}^{2}-983 E_{n+3}^{2}+208 E_{n+2}^{2}-1127 E_{n+1}^{2}+526 E_{n+4} E_{n+3}-328 E_{n+4} E_{n+2}+\right.$ $\left.382 E_{n+4} E_{n+1}-120 E_{n+3} E_{n+2}-3262 E_{n+3} E_{n+1}+1064 E_{n+2} E_{n+1}+480\right)$.

\section{References}

[1] Čerin, Z., Formulae for sums of Jacobsthal-Lucas numbers, Int. Math. Forum, 2(40), 1969-1984, 2007.

[2] Čerin, Z., Sums of Squares and Products of Jacobsthal Numbers. Journal of Integer Sequences, 10, Article 07.2.5, 2007.

[3] Chen, L., Wang, X., The Power Sums Involving Fibonacci Polynomials and Their Applications, Symmetry, 11, 2019, doi.org/10.3390/sym11050635.

[4] Frontczak, R.,Sums of powers of Fibonacci and Lucas numbers: A new bottom-up approach, Notes on Number Theory and Discrete Mathematics, 24(2), 94-103, 2018.

[5] Frontczak, R., Sums of Cubes Over Odd-Index Fibonacci Numbers, Integers, 18, 2018.

[6] Gnanam, A., Anitha, B., Sums of Squares Jacobsthal Numbers. IOSR Journal of Mathematics, 11(6), 62-64. 2015.

[7] Hathiwala, G. S., Shah, D. V., Binet-Type Formula For The Sequence of Tetranacci Numbers by Alternate Methods, Mathematical Journal of Interdisciplinary Sciences 6 (1), 37-48, 2017.

[8] Kiliç, E., Taş̧̧i, D., The Linear Algebra of The Pell Matrix, Boletín de la Sociedad Matemática Mexicana, 3(11), 2005.

[9] Kılıc, E., Sums of the squares of terms of sequence $\left\{u_{n}\right\}$, Proc. Indian Acad. Sci. (Math. Sci.) 118(1), $27-41,2008$.

[10] Melham, R. S., Some Analogs of the Identity $F_{n}^{2}+F_{n+1}^{2}=F_{2 n+1}^{2}$, Fibonacci Quarterly, 305-311, 1999.

[11] Natividad, L. R., On Solving Fibonacci-Like Sequences of Fourth, Fifth and Sixth Order, International Journal of Mathematics and Computing, 3 (2), 2013.

[12] Prodinger, H., Sums of Powers of Fibonacci Polynomials, Proc. Indian Acad. Sci. (Math. Sci.), 119(5), 567-570, 2009.

[13] Prodinger, H., Selkirk, S.J., Sums of Squares of Tetranacci Numbers: A Generating Function Approach, 2019, http://arxiv.org/abs/1906.08336v1.

[14] Raza, Z., Riaz, M., Ali, M.A., Some Inequalities on the Norms of Special Matrices with Generalized Tribonacci and Generalized Pell-Padovan Sequences, arXiv, 2015, http://arxiv.org/abs/1407.1369v2

[15] Schumacher, R., How to sum the squares of the Tetranacci numbers and the Fibonacci m-step numbers. Fibonacci Quarterly, $57: 168-175,2019$.

[16] Singh, B., Bhadouria, P., Sikhwal, O., Sisodiya, K., A Formula for Tetranacci-Like Sequence, Gen. Math. Notes, 20 (2), 136-141, 2014.

[17] Soykan, Y., A Study of Generalized Fourth-Order Pell Sequences, Journal of Scientific Research and Reports, 25(1-2), 1-18, 2019.

[18] Soykan, Y. Closed Formulas for the Sums of Squares of Generalized Fibonacci Numbers, Asian Journal of Advanced Research and Reports, 9(1), 23-39, 2020. https://doi.org/10.9734/ajarr/2020/v9i130212

[19] Soykan Y., Closed Formulas for the Sums of Cubes of Generalized Fibonacci Numbers: Closed Formulas of and $\sum_{k=0}^{n} W_{k}^{3}$ and $\sum_{k=1}^{n} W_{-k}^{3}$, Archives of Current Research International, 20(2), 58-69, 2020. DOI: 10.9734/ACRI/2020/v20i230177

[20] Soykan, Y., A Closed Formula for the Sums of Squares of Generalized Tribonacci numbers, Journal of Progressive Research in Mathematics, 16(2), 2932-2941, 2020.

[21] Soykan, Y., A Study On Sums of Cubes of Generalized Fibonacci Numbers: Closed Formulas of $\sum_{k=0}^{n} x^{k} W_{k}^{3}$ and $\sum_{k=1}^{n} x^{k} W_{-k}^{3}$, Preprints 2020, 2020040437 (doi: 10.20944/preprints202004.0437.v1).

[22] Soykan Y., On Sums of Cubes of Generalized Fibonacci Numbers: Closed Formulas of $\sum_{k=0}^{n} k W_{k}^{3}$ and $\sum_{k=1}^{n} k W_{-k}^{3}$, Asian Research Journal of Mathematics, 16(6), 37-52, 2020. DOI: 10.9734/ARJOM/2020/v16i630196 
[23] Soykan, Y., On Generalized 4-primes Numbers, submitted.

[24] N.J.A. Sloane, The on-line encyclopedia of integer sequences, http://oeis.org/

[25] Waddill, Another Generalized Fibonacci Sequence, M. E., Fibonacci Quarterly., 5 (3), 209-227, 1967.

[26] Waddill, M. E., The Tetranacci Sequence and Generalizations, The Fibonacci Quarterly, 9-20, 1992.

[27] Wamiliana., Suharsono., Kristanto, P. E., Counting the sum of cubes for Lucas and Gibonacci Numbers, Science and Technology Indonesia, 4(2), 31-35, 2019. 